\title{
Proficiency Test for Human Papillomavirus Genotyping in China
}

\author{
Rui Zhang ${ }^{\mathrm{a}, \mathrm{b}}$ Jie Huang ${ }^{\mathrm{c}}$ Pu Wang ${ }^{\mathrm{d}}$ Yu Sun ${ }^{\mathrm{a}}$ Kuo Zhang $^{\mathrm{a}}$ Jiehong Xie $^{\mathrm{a}}$ \\ Shangxian Gao ${ }^{c}$ Lunan Wang ${ }^{a}$ Jinming Li $^{a}$ \\ ${ }^{a}$ National Center for Clinical Laboratories, Beijing Hospital of the Ministry of Health, ${ }^{b}$ Graduate School, Peking Union \\ Medical College, Chinese Academy of Medical Sciences and ' Department of In Vitro Diagnostic Reagents, National \\ Institute for Food and Drug Control, Beijing, and d Department of Biochemical and Molecular Biology, School of \\ Basic Medical, Beihua University, Jilin, PR China
}

\section{Key Words}

Human papillomavirus · Genotyping · Proficiency test .

Quality control

\begin{abstract}
Background: In China, various assays for human papillomavirus (HPV) genotyping are currently used for cervical cancer screening. However, a proficiency test system is not available for standardizing and evaluating assay performance. The aim of this study was to evaluate the performance of clinical laboratories for their ability to discriminate 9 HPV types with the proficiency panel. Methods: The panel of 24 samples included cloned genomic DNAs for HPV types 6, 11, $16,18,31,33,39,51$ and 52 at different concentrations, which were distributed to 76 clinical laboratories. Results reported by participants were compared with the reference results. Results: The samples containing $10^{6} \mathrm{IU} \mathrm{HPV}-16 / \mathrm{ml}$ and $10^{6} \mathrm{IU} \mathrm{HPV}-18 / \mathrm{ml}$ were (98.7 and $96.0 \%$, respectively) identified correctly most often. For other high-risk HPV types, about $90 \%$ of data sets correctly identified samples containing $10^{6} \mathrm{GE} / \mathrm{ml}$ of HPV-6, HPV-31, HPV-33 and HPV-52, while HPV-51, HPV-11 and HPV-39 in $10^{6} \mathrm{GE} / \mathrm{ml}$ were correctly identified by only $42.7,55.6$ and $21.3 \%$ of laboratories, respectively. Conclusion: Our proficiency test system pro-
\end{abstract}

(C) 2013 S. Karger AG, Basel

0300-5526/13/0565-0295\$38.00/0 vided a traceable panel and showed that the differences in performance between laboratories were high, indicating that it is necessary for the laboratories to improve their operation and standardization of HPV genotyping.

Copyright $\odot 2013$ S. Karger AG, Basel

\section{Introduction}

Persistent human papillomavirus (HPV) infection is responsible for the majority of cervical cancers and some genital warts [1]. HPV-16, HPV-18, HPV-31, HPV-33, HPV-35, HPV-39, HPV-45, HPV-51, HPV-52, HPV-56, HPV-58, HPV-59 and HPV-68 were classified as highrisk (HR) types and associated with cervical cancer, while HPV-6 and HPV-11 were classified as low-risk (LR) types $[2,3]$. The most important HR types, HPV-16 and HPV18 , are responsible for approximately $70 \%$ of cervical cancers worldwide [4-6]. HPV types 6 and 11 are associated with most cases of anogenital warts [7, 8]. HPV genotyping is associated with evaluation and monitoring of HPV vaccination $[9,10]$. The methods used for HPV DNA typing detection can be divided into 3 types according to their typing ranges [11, 12]: (1) screening for some HPV types in aggregate, such as hybrid capture and its latest

\section{KARGER}

E-Mail karger@karger.com

www.karger.com/int 
version, HC II (Qiagen, Hilden, Germany) [13]; (2) HPV genotyping, such as linear array genotyping test (Roche, Basel, Switzerland) [14], which targets $37 \mathrm{HR}$ and LR types, and (3) HPV-16 and HPV-18 genotyping, such as Cervista HPV-16/18 (Hologic, Danbury, Conn., USA) [15], which targets HPV-16 and HPV-18. However, there are some problems with reagents and laboratory performance, such as cross-reactions, false negatives, and contamination. The trueness and limit of detection can be evaluated using certified reference material or plasmids containing the full HPV genome. Additionally, clinical laboratories should participate in external quality assessments and regularly conduct internal quality control to ensure the quality of HPV tests in their labs. In order to standardize the methodology and to evaluate assay performance, the World Health Organization (WHO) established the International Standard of HPV-16 and HPV18 DNA in 2010 and evaluated more than 21 HPV genotyping assays based on a panel containing the genomes of 14 oncogenic HPV types and 2 benign HPV types, traceable to international standards $[16,17]$.

In China, a range of assays are currently available for detecting HR HPV and identifying genotypes important in cervical cancer screening and epidemiologic research of HPV infection. However, in general, there are at least 2 features for HPV screening tests in China that are different from those reported by international studies. First, depending on different regions, the 2 most common types in China were reported to be HPV-16 or HPV-52 [1821], instead of HPV-16 and HPV-18. According to epidemiologic reports, the prevalence of HPV-16 and HPV-18 in China was lower, ranging from 1.7 to $2.5 \%$ and 0.3 to $0.8 \%$ [18-21]. In the contrast, a meta-analysis [22] on HPV detection and genotyping studies published between 1995 and 2009 showed that the worldwide prevalence of HPV-16 and HPV-18 was 3.2 and 1.4\%. Considering the various HPV genotyping methods used in the studies, the epidemiological difference might be due to the regional difference, since the meta-analysis also showed more common prevalence of HPV-16 and HPV18 in Europe, Africa and Northern America than in Asia. Other HPV types, such as HPV-6, HPV-11, HPV-31, HPV-33, HPV-39, HPV-51 and HPV-58, were also reported in different studies, while the prevalence of these types was different, depending on various region and reports [18-21]. Second, methods used for HPV genotyping primarily included PCR flow-through hybridization, PCR-reverse dot hybridization (PCR-RDB), real-time PCR, and PCR luminex, while linear array and hybrid capture were rarely used.
In our multicenter study, a proficiency panel was prepared in 2012 and used to evaluate the various HPV-typing assays for their analytical sensitivities to 9 HPV types and for their ability to discriminate the types in samples containing single and multiple HPV types.

\section{Materials and Methods}

Sources of Panel Material

The plasmids of HPV types 11 (ATCC No. 45151D), 16 (ATCC No. 45113), 18 (ATCC No. 45152) and 52 (ATCC No. VRMC$\left.29^{\mathrm{TM}}\right)$ were purchased from the American Type Culture Collection (Manassas, Va., USA). Complete genomes of HPV other types cloned into plasmid vectors were from the clinical samples and provided to the National Center for Clinical Laboratories by the respective proprietors, including Genetel Pharmaceuticals Co. (Shenzhen, China) (HPV types 6 and 31) and BGI (Beijing Genomics Institute in Beijing) (HPV types 33, 39 and 51). The HPV genomes of nearly 7.9 bp are cloned into different cloning vectors: HPV -6 in PUC 19 at position 7712, HPV-31 in PUC 19 at position 7756, HPV-33 in PBR322 at position 7881, HPV-39 in PBR322 at position 7842, and HPV-51 in PBR322 at position 7557. The HPV genomes were sequenced by BGI and sequence data was aligned to HPV reference strain sequences from GenBank. Similarities were determined using the nucleotide Basic Local Alignment Search Tool (BLAST) and the HPV types were verified.

\section{Panel Composition and Production}

Panels were prepared by the National Center for Clinical Laboratories from purified plasmids and human placental DNA. Each panel contained 23 positive samples and 1 negative sample, which were prepared by diluting the HPV recombinant DNA plasmid stock solution in the background of human placental DNA. First, the DNA of each HPV type genome was generated using an overnight culture of transformed Escherichia coli. Purified plasmids containing viral DNAs for HPV types were prepared using the Qiagen Midi-prep kit and optical density measurements were made at 260 and $280 \mathrm{~nm}$ to estimate the purity of the preparation. The plasmid concentration was determined and adjusted based on optical density using a Biophotometer (Eppendorf, Hamburg, Germany) until the final concentration is equivalent to $10^{11}$ genome equivalents/ $\mu \mathrm{l}(\mathrm{GE} / \mu \mathrm{l})$, which was calculated according to different cloning vectors and the inserted fragment of HPV genomes. Then, the plasmids were diluted to a stock concentration of $10^{8} \mathrm{GE} / \mu \mathrm{l}$ in TE buffer (10 mM Tris, $1 \mathrm{~mm}$ EDTA, pH 8.0). Human placenta DNA (Roche; Cat No. 11691112001) at a concentration of $3 \mathrm{ng} / \mu \mathrm{l}$ was added to the TE buffer to mimic human placenta DNA background. Stock samples of HPV-16 and HPV-18 were tested in 10-fold serial dilutions in TE-placenta buffer in parallel with international standards for HPV-16 (06/202) and HPV$18(06 / 206)$ distributed by the National Institute for Biological Standards and Control (Hertfordshire, UK). A real-time PCR assay was used to establish the amounts in international units by tracing the plasmids of HPV-16 and HPV-18 to the International Standard. Since international standards for HPV other types were not available, plasmids of other types were diluted according to the concentration calculated using absorbance at $260 \mathrm{~nm}$ compared 
Table 1. HPV DNA proficiency panel composition and the performance of participants and assays

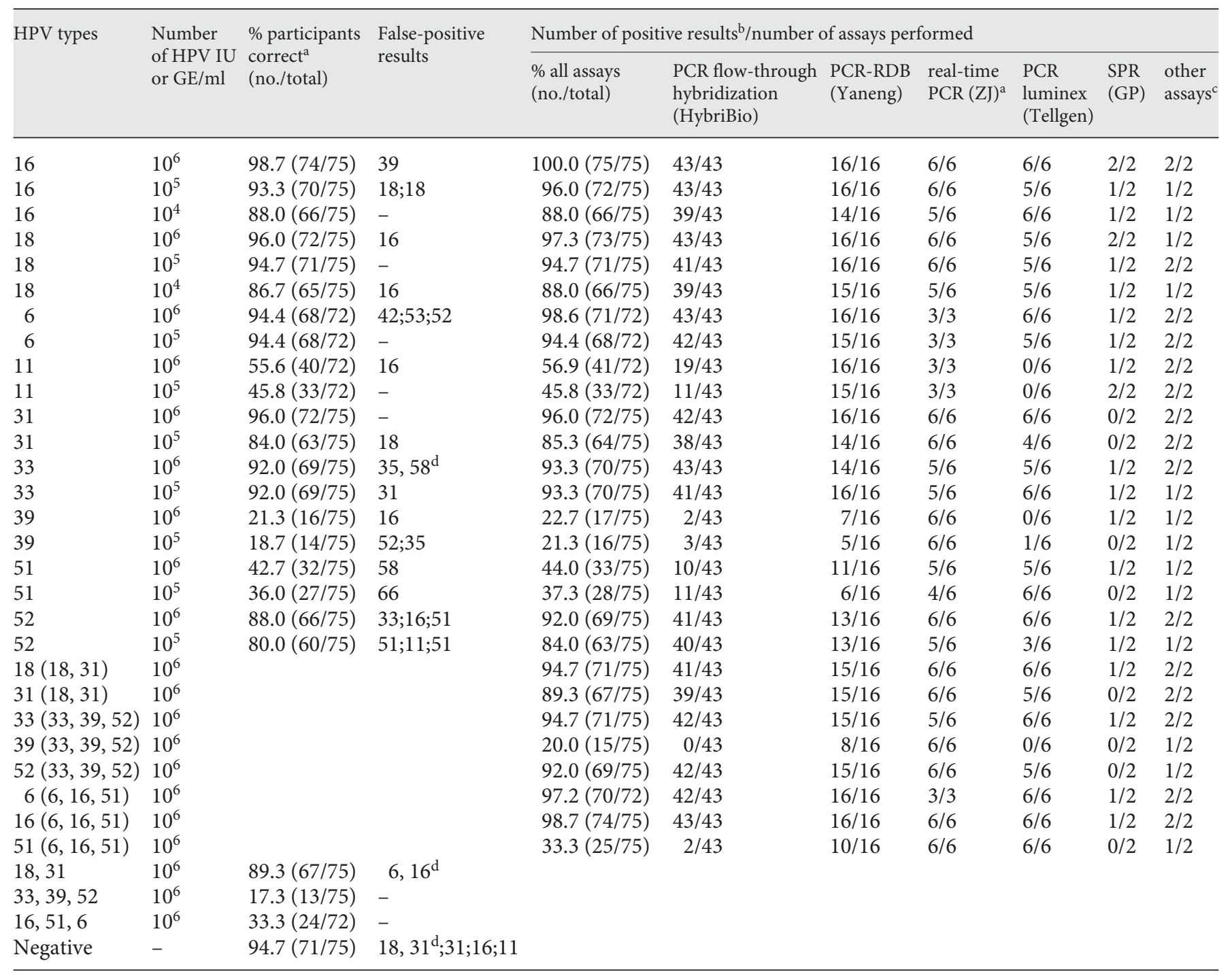

${ }^{a}$ Participants detecting the correct type as claimed with no false-positive type detected. ${ }^{b}$ Number of data sets detecting the correct type with or without false-positive results. ${ }^{c}$ Other assays included Yishengtang PCR-RDB and Taipu PCR-RDB, used by one laboratory each. ${ }^{\mathrm{d}}$ The 2 false-positive types were from the same dataset.

with the corresponding values of HPV-16 and HPV-18 plasmids. According to the criteria agreed upon at a WHO meeting regarding the standardization of HPV assays, a test should detect at least 50 IU per $5 \mu$ of HPV-16 and HPV-18 and detect at least $500 \mathrm{GE}$ per $5 \mu \mathrm{l}$ of the other HPV types included in both single and multiple infections. For this purpose, the panel was designed to contain a single infection and multiple infections at different concentrations and each plasmid was diluted to designed concentration with the TE-placenta buffer. After producing the samples, each preparation was dispensed in $0.5-\mathrm{ml}$ aliquots at the appropriate dilution and stored at $-70^{\circ} \mathrm{C}$ until shipment to the participants. The panel composition is summarized in table 1 .

\section{Participants and Data Analysis}

In total, 76 clinical laboratories were invited to participate in the study in 2012. All laboratories were assigned the samples with the same code and required to detect the samples using their routine procedures. A detailed instruction for the panel was provided to the laboratories, such as the storage condition, the assay procedure and so on. It was emphasized especially that the samples were purified DNA and should not be extracted. Questionnaires were also sent to obtain information regarding the procedures employed, including the methods, reagents, and typing results. Simultaneously, the laboratories were required to list the types that could be detected using their assays. Assays utilized by the participants 
Table 2. Proficiency results of each assay

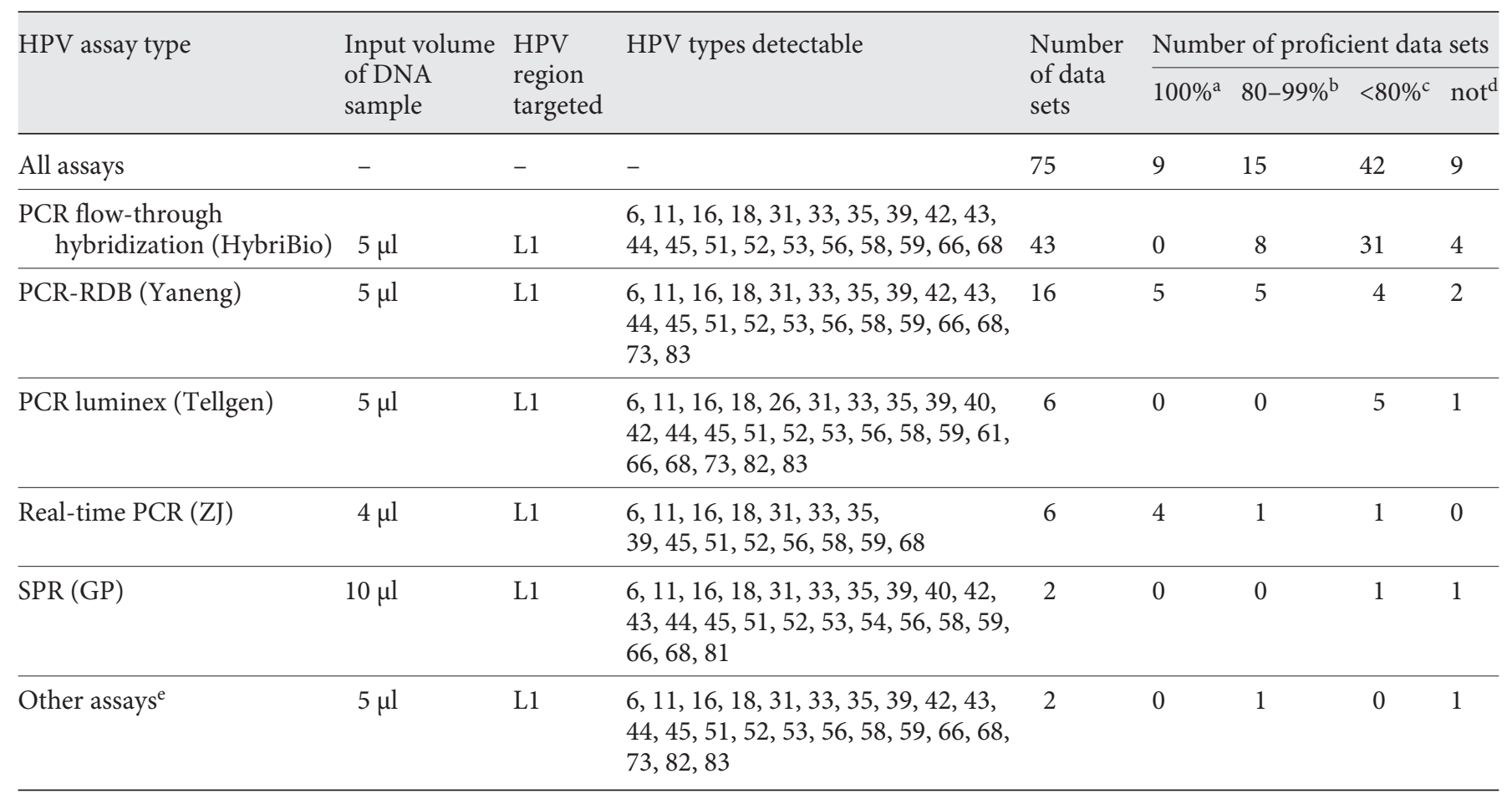

a $100 \%$ proficient: all the samples detected correctly based on the criteria. ${ }^{\text {b }} 80-99 \%$ proficient: $80-99 \%$ of the samples detected correctly based on the criteria. ${ }^{\mathrm{c}}<80 \%$ proficient: $60-80 \%$ of the samples detected correctly based on the criteria. ${ }^{\mathrm{d}}$ Not proficient: less than $60 \%$ of the samples detected correctly based on the criteria. ${ }^{e}$ Other assays included Yishengtang PCR-RDB and Taipu PCR-RDB, used by one laboratory each.

and the corresponding manufacturers, methods, and HPV types detectable have been summarized in table 2. Laboratories were asked to submit the results online within 4 weeks of receipt of the specimens. Results reported by participants were compared with the relevant reference results. Criteria used for considering a data set to be proficient were similar to the global proficiency study of HPV genotyping reported by the WHO HPV LabNet $[16,17,23]$. At most, 1 false-positive result was acceptable. The data sets reporting results only as HR or LR HPV were not included in the overall data analyses.

\section{Results}

\section{Panel Distribution and Response}

All 76 laboratories reported their results before the closing date. Mean response time of the 76 laboratories and the SD is 22 and 3.2 days, respectively. One data set generated using real-time PCR that did not discriminate between specific HPV types were not included in the overall type-specific analyses presented here. The re- agents utilized by the participants and the corresponding manufacturers are listed in table 2.

The participating laboratories included clinical laboratories and diagnostic kit manufacturers. The most commonly used assay was PCR flow-through hybridization (HybriBio, Chaozhou, China), which was used in 43 of 75 laboratories (57.3\%). Other widely used assays included PCR-RDB (Yaneng, Shenzhen, China), PCR luminex (Tellgen, Shanghai, China), and ZJ real-time PCR (Zhijiang, Shanghai, China). As the samples in the panel included plasmids of common HPV types, nearly all tests performed by laboratories should be able to detect and discriminate between types in the proficiency panel. The participating laboratories that did not identify all HPV types included 6 laboratories using ZJ real-time PCR. ZJ real-time PCR involves 2 reagents to detect $H R$ and $L R$ types. The assay for LR types only detects types 6 and 11, but it cannot distinguish between the 2 types. Therefore, laboratories using ZJ real-time PCR reported only type 6 or 11 rather than the specific type for the samples contain- 
ing either plasmid. For these laboratories, the results of type $6 / 11$ were considered acceptable. Three laboratories using ZJ real-time PCR do not detect LR types in their routine work. Therefore, only 72 results were generated for the samples containing plasmids of either type 6 or type 11 .

\section{Performance of HPV Assays}

The samples containing $10^{6} \mathrm{IU} \mathrm{HPV}-16 / \mathrm{ml}$ and $10^{6} \mathrm{IU}$ HPV $-18 / \mathrm{ml}$ were most often correctly identified (98.7 and $96.0 \%$, respectively) (table 1). For other HR HPV types, about $90 \%$ of data sets identified samples containing $10^{6} \mathrm{GE} / \mathrm{ml}$ of HPV $-6,-31,-33$, and -52 correctly, while HPV -51 in $10^{6} \mathrm{GE} / \mathrm{ml}$ was correctly identified by only $42.7 \%$ of the data sets. HPV-11 and HPV-39 were correctly identified by 55.6 and $21.3 \%$ of laboratories, respectively. Although $96.0 \%$ of laboratories reported correct results for HPV-18 and HPV-31, only $89.3 \%$ of data sets were correctly reported for samples containing multiple HPV types, including HPV-18 and HPV-31. The sample containing multiple HPV types of HPV-33, HPV-39, and HPV-52 was identified correctly by the lowest (17.3\%) number of laboratories.

The false-positive HPV types reported by participating laboratories are shown in table 1. Eighteen of the 75 laboratories had false-positive results in 28 samples. Among the 18 laboratories, 4 laboratories reported false-positive results in 3 samples and 2 laboratories reported false-positive results for HPV-16 and HPV-51 in 2 samples, respectively. Laboratories using PCR flow-through hybridization, PCR-RDB, PCR luminex, and surface plasmon resonance (Beijing GP Medical Technologies, Beijing, China) reported false-positive results in $19,5,1$ and 3 samples, respectively.

The number of HPV types detected in both single and multiple infections by different assays in the panel is shown in table 1. HPV-16 and HPV-18 could be detected at the $10^{6} \mathrm{IU} / \mathrm{ml}$ concentration by all laboratories using PCR flow-through hybridization, PCR-RDB, and ZJ realtime PCR. For HPV-6, HPV-31, HPV-33 and HPV-52, there were only $1,3,5$ and 6 data sets, respectively, which could not detect those types at $10^{6} \mathrm{GE} / \mathrm{ml}$ concentrations. In contrast, for HPV-11, HPV-39 and HPV-51, there were only 41, 17 and 33 data sets, respectively, which could detect those types at the same concentrations.

Proficiencies in detecting HPV types are shown in table 2. Based on the criteria of the global proficiency study of HPV genotyping, 9 data sets were 100\% proficient. Yaneng PCR-RDB and ZJ real-time PCR showed the highest number of proficient results (100\%). However, ZJ real-time PCR generated a higher percentage $(66.7 \%)$ of proficient results than that $(31.3 \%)$ reported by the laboratories using Yaneng PCR-RDB. Notably, except for ZJ real-time PCR, all assays had results that were not proficient.

\section{Discussion}

HPV DNA detection and typing is an essential screening parameter for detecting cervical cancer and evaluating HPV vaccines. However, a proficiency test system has not been established to evaluate the laboratory performance of HPV typing in China. In our study, recombinant plasmids containing full-length HPV DNA of types $6,11,16,18,31,33,39,51$ and 52 were used to construct a proficiency panel, since the 9 types were prevalent in China and could be detected by most of the assays for HPV genotyping.

The submitted results from laboratories showed different performances for HPV DNA-typing tests in the 9 HPV types. In the samples containing HPV-16 and HPV18 at a concentration of $10^{6} \mathrm{IU} / \mathrm{ml}, 98.7$ and $96.0 \%$ of laboratories correctly identified the types (table 1). When the concentration of plasmids of HPV-16 and HPV-18 were decreased, fewer correct data sets were generated. However, in samples containing HPV-16 and HPV-18 at a concentration of $10^{4} \mathrm{IU} / \mathrm{ml}, 88.0$ and $86.7 \%$ of data sets were reported correctly, demonstrating the high sensitivity for HPV-16 and HPV-18 tests in laboratories. Eklund et al. [17] reported that for the laboratories that participated in the global proficiency study in both 2008 and $2010,86 \%$ of laboratories could detect $10^{4} \mathrm{IU} / \mathrm{ml}$ of HPV16 in 2008, which is similar to the results in our study. However, in $201010^{4} \mathrm{IU} / \mathrm{ml}$ of HPV-16 could be detected by all the laboratories. It implicated the improvements achieved in some laboratories, which can be expected in our proficiency test in the future. Satisfying results were obtained for HPV-6, HPV-31, HPV-33 and HPV-52, as about $90 \%$ of data sets correctly identified the types in samples at a concentration of $10^{6} \mathrm{IU} / \mathrm{ml}$.

In contrast to the good performance for HPV-16, HPV-18, HPV-6, HPV-31, HPV-33 and HPV-52 typing, results for HPV-11, HPV-39 and HPV-51 reported by the laboratories were not satisfactory. HPV-11, HPV-39 and HPV -51 could not be detected at a $10^{6} \mathrm{GE} / \mathrm{ml}$ concentration by $43.1,77.3$ and $56.0 \%$ of the data sets, respectively. PCR luminex failed to detect the HPV-11 and HPV-39 plasmids in the panel, while PCR flow-through hybridization produced unsatisfying results for HPV-39 and 
HPV-51. The HPV-11, HPV-39 and HPV-51 samples were plasmids containing full-length HPV-11, HPV-39 and HPV-51 DNA, which was cloned in vectors at position far from the primer binding sites of the assays. Therefore, errors for these types might been due to primer or probe mismatch during the assays. Moreover, since the complete genome of HPV-11, HPV-39 and HPV-51 cloned into plasmid vectors were from the clinical samples, these HPV types should be detected by the assays. In epidemiologic studies examining the prevalence of HPV HR types in China, HPV-39 and HPV-51 are rarely mentioned [18-20]. It cannot be ruled out that the prevalence of HPV-39 and HPV-51 were underestimated compared to those of HPV-16, HPV-18, HPV-31, HPV-33 and HPV-52. A similar problem was noticed in the global proficiency study in 2010 [16], since for HPV-39, HPV-59 and HPV-56, there was a considerable part of laboratories that could not detect these types in the highest concentration. The laboratories and the manufacturers should be aware of the possible missing of the HPV types in the detection. In the future, more plasmids containing HPV-11, HPV-39 and HPV-51 DNA from different clinical samples might be included in the panel in order to provide more information of the assays.

Additionally, the laboratories could discriminate between each HPV type in samples with multiple HPV types, the correct data sets of which were nearly the same compared to the sample with the corresponding single HPV type (table 1). For most HPV types, the correct data sets of different concentrations were similar. Thus, the performance of HPV typing tests in laboratories primarily depends on the HPV typing abilities of the assays used, while multiple infections and virus load did not significantly influence the results.

False-positive results were another noticeable problem in our study. Eighteen of the 75 laboratories had falsepositive results in 28 samples. For each sample, there were no false-positive results for the same types in more than 3 laboratories. Therefore, false-positive results were not related to the samples in the panel. Two laboratories reported the same false-positive results for 2 samples, which were of HPV-16 and HPV-51. Obviously, the false-positive results of the 2 laboratories were caused by the PCR products of HPV-16 and HPV-51 produced in the tests performed previously. In the global proficiency study [16], there were only $71 / 132(54 \%)$ of the data sets being $100 \%$ specific, while in our study, 57/75 (76\%) of the data sets were $100 \%$ specific. One of the possible reasons of relatively low false-positive rate in our study is the mandatory rules regarding the laboratory environment and op- eration procedures for the laboratories carrying out PCR tests in China since 2002. In addition, the different sensitivities of various assays participating in the proficiency test were another possible reason. The results of the WHO proficiency test indicated that the increased sensitivity was accompanied by increased amounts of false-positive results. Therefore, the negative control is absolutely necessary in the routine work for the laboratories.

According to the criteria of the global proficiency study of HPV genotyping, 9 data sets were $100 \%$ proficient. No data sets were reported to be $100 \%$ proficient by the participating laboratories using PCR flow-through hybridization, or PCR luminex. In contrast, ZJ real-time PCR showed good performance for each HPV type included in the proficiency panel, indicating that real-time PCR detects and identifies different HPV types accurately. However, it should be emphasized that the DNA extraction step for samples in our proficiency panel could not be evaluated. Therefore, good performance in the proficiency test only represented the ability of HPV amplification and typing. However, the differences in performance of laboratories using the same HPV typing assays were large, indicating that it is necessary for laboratories to improve their operations. In addition, it should be noticed that the plasmids are not suitable for the assays if their target genes bridge the site of cloning. In this study, all the commercial assays included were directed to the L1 gene, while the HPV genomes were cloned into vectors at positions from 7500 to 7900 , which existed outside L1 region. Therefore, the constructed plasmids were suitable for all the assays in this PT. Nevertheless, it is still essential for future PT participants to provide the primer binding sites and target genes of their assays so as to evaluate the suitability of the plasmids. Besides, our proficiency panel only contained $9 \mathrm{HPV}$ types. In the future, HPV types 35, 45, 56, 58, 59, 61, 66, 67, 68, 69, 71, 73, 81 and 82 will be included to provide more valuable information for HPV genotyping in clinical laboratories.

In conclusion, our proficiency test system provided a traceable panel, which contains most of the prevalent HPV types in China and is useful for evaluating the assay performance. It was planned that the proficiency test for HPV genotyping be carried out every year to promote the comparability of the HPV genotyping results from different laboratories. It should be noticed that the samples in our panel represented an almost ideal sample setting containing HPV DNA, while real clinical specimens always contain different components that may inhibit or alter the performance of HPV tests and the presence of multiple infections with some types will also serve to bias the re- 
sults further. Nevertheless, even in this panel, many laboratories performed HPV tests poorly and reported unsatisfying results, which indicated that it is necessary for the laboratories to improve their operation and standardization of HPV genotyping.

\section{Acknowledgements}

This work was supported by a grant from the National High Technology Research and Development Program of China (863 Program) (No. 2011AA02A116).

\section{References}

1 Bulkmans NW, Berkhof J, Rozendaal L, van Kemenade FJ, Boeke AJ, Bulk S, Voorhorst FJ, Verheijen RH, van Groningen K, Boon ME, Ruitinga W, van Ballegooijen M, Snijders PJ, Meijer CJ: Human papillomavirus DNA testing for the detection of cervical intraepithelial neoplasia grade 3 and cancer: 5-year followup of a randomised controlled implementation trial. Lancet 2007;370:1764-1772.

2 Muñoz N, Bosch FX, de Sanjosé S, Herrero R, Castellsagué X, Shah KV, Snijders PJ, Meijer CJ, International Agency for Research on Cancer Multicenter Cervical Cancer Study Group: Epidemiologic classification of human papillomavirus types associated with cervical cancer. N Engl J Med 2003;348:518527.

- 3 Smith JS, Lindsay L, Hoots B, Keys J, Franceschi S, Winer R, Clifford GM: Human papillomavirus type distribution in invasive cervical cancer and high-grade cervical lesions: a meta-analysis update. Int J Cancer 2007;121: 621-632.

-4 Bosch FX, Burchell AN, Schiffman M, Giuliano AR, de Sanjose S, Bruni L, Tortolero-Luna G, Kjaer SK, Muñoz N: Epidemiology and natural history of human papillomavirus infections and type-specific implications in cervical neoplasia. Vaccine 2008;26(suppl 10):K1-K16.

5 Li N, Franceschi S, Howell-Jones R, Snijders PJ, Clifford GM: Human papillomavirus type distribution in 30,848 invasive cervical cancers worldwide: Variation by geographical region, histological type and year of publication. Int J Cancer 2011;128:927-935.

6 Seme K, Fujs K, Kocjan BJ, Poljak M: Resolving repeatedly borderline results of Hybrid Capture 2 HPV DNA Test using polymerase chain reaction and genotyping. J Virol Methods 2006;134:252-256.

7 Ault KA: Epidemiology and natural history of human papillomavirus infections in the female genital tract. Infect Dis Obstet Gynecol 2006;2006(suppl):40470.
8 Vandepapeliere P, Barrasso R, Meijer CJ, Walboomers JM, Wettendorff M, Stanberry LR, Lacey CJ: Randomized controlled trial of an adjuvanted human papillomavirus (HPV) type 6 L2E7 vaccine: infection of external anogenital warts with multiple HPV types and failure of therapeutic vaccination. J Infect Dis 2005; 192:2099-2107.

-9 Kulasingam SL, Myers ER: Potential health and economic impact of adding a human papillomavirus vaccine to screening programs. JAMA 2003;290:781-789.

10 Johnson AM, Mercer CH, Beddows S, de Silva N, Desai S, Howell-Jones R, Carder C, Sonnenberg P, Fenton KA, Lowndes C, Soldan K: Epidemiology of, and behavioural risk factors for, sexually transmitted human papillomavirus infection in men and women in Britain. Sex Transm Infect 2012;88:212-217.

11 Poljak M, Kocjan BJ: Commercially available assays for multiplex detection of alpha human papillomaviruses. Expert Rev Anti Infect Ther 2010;8:1139-1162.

12 Kroupis C, Vourlidis N: Human papilloma virus (HPV) molecular diagnostics. Clin Chem Lab Med 2011;49:1783-1799.

13 Dufresne S, Sauthier P, Mayrand MH, Petignat $\mathrm{P}$, Provencher D, Drouin P, Gauthier P, Dupuis MJ, Michon B, Ouellet S, Hadjeres R, Ferenczy A, Franco EL, Coutlée F: Human papillomavirus (HPV) DNA triage of women with atypical squamous cells of undetermined significance with Amplicor HPV and Hybrid Capture 2 assays for detection of high-grade lesions of the uterine cervix. J Clin Microbiol 2011;49:48-53.

14 Papachristou E, Sypsa V, Paraskevis D, Gkekas A, Politi E, Nicolaidou E, Anifantis I, Psichogiou M, Tsitsika A, Hadjivassiliou M, Petrikkos G, Katsambas A, Creatsas G, Hatzakis A: Prevalence of different HPV types and estimation of prognostic risk factors based on the linear array HPV genotyping test. J Med Virol 2009;81:2059-2065.

15 Einstein MH, Martens MG, Garcia FA, Ferris DG, Mitchell AL, Day SP, Olson MC: Clinical validation of the Cervista HPV HR and 16/18 genotyping tests for use in women with ASC-US cytology. Gynecol Oncol 2010;118: $116-122$.
16 Eklund C, Forslund O, Wallin KL, Zhou T, Dillner J, WHO Human Papillomavirus Laboratory Network: The 2010 global proficiency study of human papillomavirus genotyping in vaccinology. J Clin Microbiol 2012;50:2289-2298.

$\checkmark 17$ Eklund C, Zhou T, Dillner J, WHO Human Papillomavirus Laboratory Network: Global proficiency study of human papillomavirus genotyping. J Clin Microbiol 2010;48:4147-4155.

18 Zhao R, Zhang WY, Wu MH, Zhang SW, Pan J, Zhu L, Zhang YP, Li H, Gu YS, Liu XZ: Human papillomavirus infection in Beijing, People's Republic of China: a population-based study. Br J Cancer 2009;101:1635-1640.

19 Wang S, Wei H, Wang N, Zhang S, Zhang Y, Ruan Q, Jiang W, Xiao Q, Luan X, Qian X, Zhang L, Gao X, Sun X: The prevalence and role of human papillomavirus genotypes in primary cervical screening in the northeast of China. BMC Cancer 2012;12:160.

20 Ye J, Cheng X, Chen X, Ye F, Lü W, Xie X: Prevalence and risk profile of cervical Human papillomavirus infection in Zhejiang Province, southeast China: a population-based study. Virol J 2010;7:66.

21 Chen Q, Xie LX, Qing ZR, Li LJ, Luo ZY, Lin $M$, Zhang SM, Chen WZ, Lin BZ, Lin QL, Li $\mathrm{H}$, Chen WP, Zheng PY, Mao LZ, Chen CY, Yang C, Zhan YZ, Liu XZ, Zheng JK, Yang LY: Epidemiologic characterization of human papillomavirus infection in rural Chaozhou, eastern Guangdong Province of China. PLoS One 2012;7:e32149.

22 Bruni L, Diaz M, Castellsagué X, Ferrer E, Bosch FX, de Sanjosé S: Cervical human papillomavirus prevalence in 5 continents: metaanalysis of 1 million women with normal cytological findings. J Infect Dis 2010;202:17891799.

23 Ferguson M, Wilkinson DE, Zhou T: WHO meeting on the standardization of HPV assays and the role of the WHO HPV Laboratory Network in supporting vaccine introduction held on 24-25 January 2008, Geneva, Switzerland. Vaccine 2009;27:337-347. 